\title{
BIRATIONALITY OF THE TANGENT MAP FOR MINIMAL RATIONAL CURVES*
}

\author{
JUN-MUK HWANG ${ }^{\dagger}$ AND NGAIMING MOK ${ }^{\ddagger}$
}

Dedicated to Professor Yum-Tong Siu on his sixtieth birthday

\begin{abstract}
For a uniruled projective manifold, we prove that a general rational curve of minimal degree through a general point is uniquely determined by its tangent vector. As applications, among other things we give a new proof, using no Lie theory, of our earlier result that a holomorphic map from a rational homogeneous space of Picard number 1 onto a projective manifold different from the projective space must be a biholomorphic map.
\end{abstract}

1. Introduction. Let $X$ be an irreducible uniruled projective variety. Let $\operatorname{RatCurves}^{n}(X)$ be the normalized space of rational curves on $X$ in the sense of [Ko]. For an irreducible component $\mathcal{K}$ of $\operatorname{RatCurves}^{n}(X)$, let $\rho: \mathcal{U} \rightarrow \mathcal{K}$ and $\mu: \mathcal{U} \rightarrow X$ be the associated universal family morphisms. In other words, $\rho$ is a $\mathbb{P}^{1}$-bundle over $\mathcal{K}$ and for $\alpha \in \mathcal{K}$, the corresponding rational curve in $X$ is $\mu\left(\rho^{-1}(\alpha)\right)$. An irreducible component $\mathcal{K}$ of $\operatorname{RatCurves}^{n}(X)$ is a minimal component if $\mu$ is dominant and for a general point $x \in X, \mu^{-1}(x)$ is projective. Members of a minimal component are called minimal rational curves. For example, rational curves passing through a general point of $X$ of minimal degree with respect to a fixed ample line bundle are minimal rational curves. Denote by $\mathbb{P} T(X)$ the projectivization of the tangent bundle of the smooth part of $X$. Given a minimal component $\mathcal{K}$, consider the rational map

$$
\tau: \mathcal{U} \rightarrow \mathbb{P} T(X)
$$

defined by

$$
\tau(\alpha):=\mathbb{P} T_{x}(C)
$$

for $\alpha \in \mathcal{U}$ such that $x:=\mu(\alpha)$ is a smooth point of $X$ and $\rho(\alpha)$ corresponds to a rational curve $C$ on $X$ smooth at $x$. Let $\mathcal{C} \subset \mathbb{P} T(X)$ be the proper image of $\tau$. We call $\tau$ the tangent map of $\mathcal{K}$ and $\mathcal{C}$ the total variety of minimal rational tangents of $\mathcal{K}$.

When $X$ is smooth, it is well-known that the degree of a minimal rational curve with respect to the anti-canonical bundle is bounded by $\operatorname{dim}(X)+1$ and the tangent map $\tau: \mathcal{U} \rightarrow \mathcal{C}$ is generically finite. We will prove the following.

THEOREM 1. For any uniruled projective manifold $X$ and any minimal component $\mathcal{K}$, the tangent map $\tau: \mathcal{U} \rightarrow \mathcal{C}$ is birational.

In the process of proving Theorem 1, we will also prove

THEOREM 2. Suppose a uniruled projective manifold has two distinct minimal components $\mathcal{K}$ and $\mathcal{K}^{\prime}$. Then their total spaces of minimal rational tangents $\mathcal{C}$ and $\mathcal{C}^{\prime}$ are distinct.

* Received April 7, 2003; accepted for publication August 26, 2003.

$\dagger$ Korea Institute for Advanced Study, 207-43 Cheongryangri-dong, Seoul 130-722, Korea (jmhwang@ns.kias.re.kr). Supported by the Korea Research Foundation Grant (KRF-2002-070C00003).

¥Department of Mathematics, The University of Hong Kong, Pokfulam Road, Hong Kong (nmok@hkucc.hku.hk). Supported by a CERG of the Research Grants Council of Hong Kong. 
Theorem 1 and Theorem 2 imply that a general rational curve of minimal degree through a general point of $X$ is uniquely determined by its tangent vector. Theorem 1 is proved under some special assumptions on $\mathcal{K}$, which do not always hold, in [KK]. Their method is completely different from ours.

In addition to its intrinsic interest, Theorem 1 has several interesting consequences. For a point $x \in X$, the subvariety $\mathcal{C}_{x}=\mathcal{C} \cap \mathbb{P} T_{x}(X)$ is called the variety of minimal rational tangents at $x$. It is known that $\mu^{-1}(x)$ is a (not necessarily irreducible) smooth projective variety for a general point $x$. Kebekus showed that for a general $x$, the restriction of the tangent map to the fiber at $x, \tau_{x}: \mu^{-1}(x) \rightarrow \mathcal{C}_{x}$ is a finite morphism ([Ke, Theorem 3.4]). Thus Theorem 1 has the following consequence.

COROLlary 1. For any uniruled projective manifold and a minimal component, the normalization of the variety of minimal rational tangents at a general point is smooth.

This has an application to the rigidity of generically finite morphisms to Fano manifolds of Picard number 1 . We say that $\mathcal{C}_{x}$ is non-linear, if one (hence all) of the components of $\mathcal{C}_{x}$ is not a linear subvariety of $\mathbb{P T}_{x}(X)$. We will prove

Theorem 3. Let $\chi: \mathcal{X} \rightarrow \Delta:=\{t \in \mathbb{C},|t|<1\}$ be a regular family of Fano manifolds of Picard number 1 so that $X_{0}=\chi^{-1}(0)$ has a minimal component whose variety of minimal rational tangents at a general point is non-linear. For a given projective manifold $X^{\prime}$, suppose there exists a surjective morphism $f: \mathcal{X}^{\prime}=X^{\prime} \times \Delta \rightarrow$ $\mathcal{X}$ respecting the projections to $\Delta$ so that $f_{t}: X^{\prime} \rightarrow X_{t}$ is a generically finite morphism for each $t \in \Delta$. Then there exists $\epsilon>0$ and a holomorphic family of biholomorphic morphisms $v_{t}: X_{0} \rightarrow X_{t}$ for $|t|<\epsilon$, satisfying $v_{0}=\operatorname{Id}$ and $f_{t}=v_{t} \circ f_{0}$.

One interesting consequence is the following.

THEOREM 4. Let $G / P$ be a rational homogeneous space of Picard number 1 . If $f: G / P \rightarrow X$ is a surjective morphism to a smooth projective variety $X$ of positive dimension, then either $X \cong \mathbb{P}^{n}, n=\operatorname{dim}(G / P)$, or $f$ is a biregular morphism.

This was originally proved in [HM1] by using quite a bit of Lie theory. The proof given here uses no Lie theory.

A natural question arising from Theorem 3 is to understand when a projective manifold $X$ admits a minimal component $\mathcal{K}$ whose variety of minimal rational tangents at a general point is non-linear. We expect the following.

Conjecture. For any Fano manifold of Picard number 1 excepting the projective space and for any minimal component, the variety of minimal rational tangents at a general point is non-linear if its dimension is positive. In particular, for a Fano manifold of Picard number 1 with index $>2$ excepting the projective space, any minimal component has non-linear varieties of minimal rational tangents at general points.

There are two ingredients of the proof of Theorem 1, the work of Cho-MiyaokaShepherd-Barron ([CMS]) and the theory of differential systems on the subvariety of the projectivized tangent bundle. Roughly speaking, [CMS] proves Theorem 1 in the special case when $\tau: \mathcal{U} \rightarrow \mathbb{P} T(X)$ is dominant and the theory of differential systems reduces the general case to this special case. The theory of differential systems we need will be explained in Section 2. Theorem 1 and Theorem 2 will be proved in Section 3. Theorem 3 and Theorem 4 will be proved in Section 4 . We will work over 
the field of complex numbers. Unless otherwise stated, the topology considered is the analytic topology.

2. Differential systems on the subvariety of the projectivized tangent bundle. We need to study some natural distributions defined on a subvariety of the projectivized tangent bundle of a complex manifold. Since this theory does not seem to be well-known to algebraic geometers or complex analysts, we will give a full account in this section. In the standard literature, a distribution on a complex manifold means a subbundle of the tangent bundle. In this article, it will be used in a broader sense.

A distribution on an irreducible normal variety is a subbundle of the tangent bundle of a Zariski open subset of the smooth part of the normal variety. This Zariski open subset will be called the domain of definition of the distribution. For simplicity, we will regard two distributions identical if they agree on the intersection of their domains of definition. Thus, given any distribution on a normal variety $X$, there is a smallest subvariety $S$ containing the singular locus of $X$ such that the distribution can be identified with a distribution defined on $X-S$. We will call $S$ the singular locus of the distribution.

By taking local holomorphic sections, we can view a distribution $\mathcal{V}$ as a locally free sheaf on the domain of definition. The Lie bracket of local vector fields define a $\mathbb{C}$-linear sheaf map [,] $: \mathcal{V} \times \mathcal{V} \rightarrow \Theta$ where $\Theta$ denotes the tangent sheaf of the domain of definition. The distribution defined by $[\mathcal{V}, \mathcal{V}]+\mathcal{V}$ is called the first derived system of $\mathcal{V}$ and is denoted by $\partial \mathcal{V}$. The domain of definition of $\partial \mathcal{V}$ may be different from that of $\mathcal{V}$. The Lie bracket defines a holomorphic vector bundle morphism $\bigwedge^{2} \mathcal{V} \rightarrow \Theta / \mathcal{V}$ over the domain of definition. This section of $\operatorname{Hom}\left(\bigwedge^{2} \mathcal{V}, \Theta / \mathcal{V}\right)$ over the domain of definition will be called the Frobenius bracket tensor of $\mathcal{V}$. By abuse of notation, we will use the same notation [,] for the Frobenius bracket tensor and the Lie bracket of vector fields. By the famous Frobenius theorem, if the Frobenius bracket tensor vanishes, or equivalently, if $\partial \mathcal{V}=\mathcal{V}$, the distribution $\mathcal{V}$ is integrable and defines a foliation on its domain of definition. In this case we will use the notation $\mathcal{V}$ to mean both the distribution and the foliation defined by it. A leaf of an integrable distribution means a maximal irreducible submanifold in the domain of definition which is tangent to the given distribution. The Cauchy characteristic of $\mathcal{V}$ is the distribution $C h(\mathcal{V})$ which is defined at a general point $x$ by

$$
C h(\mathcal{V})_{x}=\left\{v \in \mathcal{V}_{x},\left[v, \mathcal{V}_{x}\right]=0\right\} .
$$

The Cauchy characteristic of any distribution is integrable, as can be easily checked using the Jacobi identity.

Let $\xi: Y \rightarrow X$ be a dominant morphism between two varieties. For a distribution $\mathcal{D}$ on $X, d \xi^{*} \mathcal{D}$ denotes the distribution on $Y$ defined by

$$
\left(d \xi^{*} \mathcal{D}\right)_{y}=\left(d \xi_{y}\right)^{-1}\left(\mathcal{D}_{\xi(y)}\right)
$$

at a general point $y \in Y$ such that the differential $d \xi_{y}: T_{y}(Y) \rightarrow T_{\xi(y)}(X)$ is surjective and $\xi(y)$ lies in the domain of definition of $\mathcal{D}$. $d \xi^{*} \mathcal{D}$ will be called the pull-back of $\mathcal{D}$ by $\xi$. Given a local section $w$ of $\mathcal{D}$ in a neighborhood of $\xi(y)$, we can find a local section $d \xi^{*} w$ of $d \xi^{*} \mathcal{D}$ in a neighborhood of $y$ so that $w=d \xi_{*}\left(d \xi^{*} w\right)$. We will say that $d \xi^{*} w$ is a lift of $w$. Two different lifts of $w$ differ by a local vector field tangent to the fibers of $\xi$. The proof of the following lemma is elementary. 
Lemma 1. For any dominant morphism $\xi: Y \rightarrow X$ and a distribution $\mathcal{D}$ on $X$,

$$
\begin{aligned}
d \xi^{*}(\partial \mathcal{D}) & =\partial\left(d \xi^{*} \mathcal{D}\right) \\
d \xi^{*} C h(\mathcal{D}) & =C h\left(d \xi^{*} \mathcal{D}\right)
\end{aligned}
$$

In particular, tangent vectors to the fibers of $\xi$ are contained in $C h\left(d \xi^{*} \mathcal{D}\right)$.

For the rest of this section, let us fix a complex manifold $X$ and an irreducible subvariety $\mathcal{C} \subset \mathbb{P} T(X)$ which is dominant over $X$. There are two naturally defined distributions $\mathcal{J}$ and $\mathcal{P}$ on $\mathcal{C}$. At a general point $\alpha \in \mathcal{C}$, they are defined by

$$
\begin{aligned}
& \mathcal{J}_{\alpha}:=(d \pi)^{-1}(\mathbb{C} \alpha) \\
& \mathcal{P}_{\alpha}:=(d \pi)^{-1}\left(\hat{T}_{\alpha}\left(\mathcal{C}_{x}\right)\right)
\end{aligned}
$$

where $d \pi: T_{\alpha}(\mathcal{C}) \rightarrow T_{x}(X)$ is the differential of the natural projection $\pi: \mathcal{C} \rightarrow X$ at $\alpha \in \mathcal{C}, x=\pi(\alpha)$, and $\hat{T}_{\alpha}\left(\mathcal{C}_{x}\right) \subset T_{x}(X)$ is the linear tangent space of $\mathcal{C}_{x}:=\pi^{-1}(x) \subset$ $\mathbb{P} T_{x}(X)$ at $\alpha$. Both $\mathcal{J}$ and $\mathcal{P}$ are canonically determined by $\mathcal{C}$. $\mathcal{J}$ has rank $p+1$ and $\mathcal{P}$ has rank $2 p+1$, where $p$ is the fiber dimension of $\pi: \mathcal{C} \rightarrow X$. Also we have the trivial vertical distribution $\mathcal{V}$ of rank $p$ on $\mathcal{C}$ defining the fibers of $\pi$. Clearly, $\mathcal{V} \subset \mathcal{J} \subset \mathcal{P}$.

Proposition 1. In a neighborhood of a general point of $\mathcal{C}$, choose a line subbundle $\mathcal{F}$ of $\mathcal{J}$ such that $\mathcal{V}+\mathcal{F}=\mathcal{J}$. Then every local section of $\mathcal{P}$ is of the form $[v, f]+u+f^{\prime}$ where $u, v$ are local sections of $\mathcal{V}$ and $f, f^{\prime}$ are local sections of $\mathcal{F}$. In other words, $\mathcal{P}=\partial \mathcal{J}$.

Proof. For notational simplicity, we will work over $\Xi:=T(X) \backslash(0$-section). Let $\xi: \Xi \rightarrow \mathbb{P} T(X)$ be the natural $\mathbb{C}^{*}$-bundle. Let $\hat{\mathcal{C}}:=\xi^{-1}(\mathcal{C})$. We will denote the restriction of $\xi$ to $\hat{\mathcal{C}}$ by the same letter $\xi$. Let $\hat{\mathcal{J}}:=d \xi^{*} \mathcal{J}, \hat{\mathcal{P}}:=d \xi^{*} \mathcal{P}, \hat{\mathcal{V}}:=d \xi^{*} \mathcal{V}$, and $\hat{\mathcal{F}}:=d \xi^{*} \mathcal{F}$ be the distributions pulled-back to $\hat{\mathcal{C}}$. By Lemma 1 , it suffices to check that $\hat{\mathcal{P}}=\partial \hat{\mathcal{J}}$.

We start with $\partial \hat{\mathcal{J}} \subset \hat{\mathcal{P}}$. It suffices to show $[\hat{\mathcal{V}}, \hat{\mathcal{F}}] \subset \hat{\mathcal{P}}$. Let $x_{1}, \ldots, x_{n}$ be a local coordinate system on $X$. Let $\lambda_{1}=d x_{1}, \ldots, \lambda_{n}=d x_{n}$ be linear coordinates in the vertical direction of $\Xi$. Let $\hat{v}=\sum_{i} v_{i} \frac{\partial}{\partial \lambda_{i}}$ be a lift of a local section of $\mathcal{V}$ and $\hat{f}=\sum_{i} f_{i} \frac{\partial}{\partial \lambda_{i}}+\zeta \sum_{j} \lambda_{j} \frac{\partial}{\partial x_{j}}$ be a lift of a local section of $\mathcal{F}$ over a small open set in $\hat{\mathcal{C}}$. Here $v_{i}, f_{i}, \zeta$ are suitable local holomorphic functions. Dividing by $\zeta$ and looking at general points outside the zero set of $\zeta$, we may assume that $\zeta \equiv 1$. Then $[\hat{v}, \hat{f}]=\sum_{i} v_{i} \frac{\partial}{\partial x_{i}}$ modulo $\hat{\mathcal{V}}$. But this is precisely the vectors $\hat{v}$ viewed as the tangent vectors to $X$. Hence $[\hat{v}, \hat{f}]$ is a local section of $\hat{\mathcal{P}}$.

From the above expression of $[\hat{v}, \hat{f}]$ modulo $\hat{\mathcal{V}}$, we see that the rank of $\partial \hat{\mathcal{J}}$ is higher than the rank of $\hat{\mathcal{J}}$ by at least $p$, which shows $\partial \hat{\mathcal{J}}=\hat{\mathcal{P}}$.

We will describe the Frobenius bracket tensor of the distribution $\mathcal{P}$ in terms of the projective geometry of $\mathcal{C}_{x}$. For this, we recall the definitions of the second fundamental form of a subvariety in the projective space.

Let $V$ be a complex vector space and $Z \subset \mathbb{P} V$ be a subvariety of the projective space. For a smooth point $x \in Z$, we are going to define a symmetric bilinear form $I I_{x, Z}: T_{x}(Z) \otimes T_{x}(Z) \rightarrow N_{x}(Z ; \mathbb{P} V)$, called the second fundamental form of $Z$ at $x$ as follows. Let $\xi: V \backslash 0 \rightarrow \mathbb{P} V$ be the natural $\mathbb{C}^{*}$-bundle. We denote $\xi^{-1}(x)$ by $\hat{x}$ and $\xi^{-1}(Z)$ by $\hat{Z}$. Let $\hat{T}_{x}(Z) \subset V$ be the affine tangent space of $Z$ at $x$ which can be 
naturally identified with the tangent space $T_{\bar{x}}(\hat{Z})$ of $\hat{Z}$ at any point $\bar{x}$ of $\hat{x}$. We have natural identifications

$$
\begin{aligned}
T_{x}(Z) & =\operatorname{Hom}\left(\hat{x}, \hat{T}_{x}(Z) / \hat{x}\right) \\
N_{x}(Z ; \mathbb{P} V) & =\operatorname{Hom}\left(\hat{x}, V / \hat{T}_{x}(Z)\right) .
\end{aligned}
$$

Given two elements $u_{o}$ and $v_{o}$ of $T_{x}(Z)$, choose local vector fields $u$ and $v$ on $Z$ with $u_{x}=u_{o}$ and $v_{x}=v_{o}$. Let $\hat{u}$ and $\hat{v}$ be their lifts in an open subset of $\hat{Z}$. We extend them to local vector fields in an open subset of $V \backslash 0$ and denote these extended vector fields by the same symbols. In terms of a linear coordinate system $x_{1}, \ldots, x_{n}$ on $V$, we can write

$$
\begin{aligned}
& \hat{u}=u_{1} \frac{\partial}{\partial x_{1}}+\cdots+u_{n} \frac{\partial}{\partial x_{n}} \\
& \hat{v}=v_{1} \frac{\partial}{\partial x_{1}}+\cdots+v_{n} \frac{\partial}{\partial x_{n}} .
\end{aligned}
$$

We define

$$
\begin{aligned}
& \hat{v}(\hat{u}):=\sum_{i, j=1}^{n} v_{i} \frac{\partial u_{j}}{\partial x_{i}} \frac{\partial}{\partial x_{j}} \\
& \hat{u}(\hat{v}):=\sum_{i, j=1}^{n} u_{i} \frac{\partial v_{j}}{\partial x_{i}} \frac{\partial}{\partial x_{j}} .
\end{aligned}
$$

These are local vector fields on $V \backslash 0$. They are not necessarily tangent to $\hat{Z}$, but

$$
\hat{v}(\hat{u})-\hat{u}(\hat{v})=[\hat{v}, \hat{u}]
$$

is a lift of the local vector field $[v, u]$ on $Z$. We denote the value of $\hat{v}(\hat{u})$ at a point $\bar{x} \in \hat{x}$ modulo $\hat{T}_{x}(Z)=T_{\bar{x}}(\hat{Z})$ by $\hat{I I}_{\bar{x}}\left(u_{o}, v_{o}\right)$. It is easy to see that this vector in $V / \hat{T}_{x}(Z)$ is independent of the choices of $u, v, \hat{u}$, or $\hat{v}$ and it depends linearly on the choice of $\bar{x} \in \hat{x}$. In other words, $\bar{x} \mapsto \hat{I I} \bar{x}_{\bar{x}}\left(u_{o}, v_{o}\right)$ defines an element of $\operatorname{Hom}\left(\hat{x}, V / \hat{T}_{x}(Z)\right)=N_{x}(Z ; \mathbb{P} V)$. This element of $N_{x}(Z ; \mathbb{P} V)$ is defined to be $I I_{x, Z}\left(u_{o}, v_{o}\right)$. From the fact that $[\hat{u}, \hat{v}]$ is tangent to $\hat{Z}$, we can see the symmetry $I I_{x, Z}\left(u_{o}, v_{o}\right)=I I_{x, Z}\left(v_{o}, u_{o}\right)$.

Another equivalent definition of the second fundamental form is via the Gauss map. The Gauss map $\gamma: Z \rightarrow G r(\operatorname{dim} Z+1 ; V)$ is a rational map assigning the affine tangent spaces to $Z$ at smooth points of $Z$. In other words, it is defined by $\gamma(x)=\left[\hat{T}_{x}(Z)\right]$ at a smooth point $x \in Z$.

The derivative of the Gauss map

$$
d \gamma_{x}: T_{x}(Z) \rightarrow T_{\left[\hat{T}_{x}(Z)\right]}(G r(\operatorname{dim} Z+1 ; V))=\operatorname{Hom}\left(\hat{T}_{x}(Z), V / \hat{T}_{x}(Z)\right)
$$

induces an element of $T_{x}(Z) \otimes T_{x}(Z) \rightarrow N_{x}(Z ; \mathbb{P} V)$ and one can check that our definition of $I I_{x, Z}$ is just an explicit coordinate description of this element. The following result is classical:

Lemma 2 ([GH, 2.10]). The closures of the fibers of the Gauss map are linear subspaces in $V$.

Now we are ready to describe the Frobenius bracket of $\mathcal{P}$ : 
Proposition 2. Let $\alpha \in \mathcal{C}$. Choose a local complement $\mathcal{F}$ as in Proposition 1 and a section $f$ of $\mathcal{F}$ near $\alpha$. Given two vectors $u_{o}$ and $v_{o}$ in $T_{\alpha}\left(\mathcal{C}_{x}\right)$ with $x=\pi(\alpha)$, let $u$ (resp. $v$ ) be a local vector field on a neighborhood of $\alpha$ in $\mathcal{C}$ tangent to fibers of $\pi$ such that $u_{\alpha}=u_{o}$ (resp. $\left.v_{\alpha}=v_{o}\right)$. Let $[v, f]_{\alpha}$ be the value of the local vector field $[v, f]$ on $\mathcal{C}$ at the point $\alpha$. Then the Frobenius bracket tensor for the distribution $\mathcal{P}$ at $\alpha$

$$
\begin{aligned}
{[,]: \bigwedge^{2} \mathcal{P}_{\alpha} } & \rightarrow T_{\alpha}(\mathcal{C}) / \mathcal{P}_{\alpha} \\
& =T_{x}(X) / \hat{T}_{\alpha}\left(\mathcal{C}_{x}\right) \\
& =\hat{\alpha} \otimes N_{\alpha}\left(\mathcal{C}_{x} ; \mathbb{P} T_{x}(X)\right)
\end{aligned}
$$

satisfies

$$
[u,[v, f]]_{\alpha}=\pi_{*} f_{\alpha} \otimes I I_{\alpha, \mathcal{C}_{x}}(u, v) \in \hat{\alpha} \otimes N_{\alpha}\left(\mathcal{C}_{x} ; \mathbb{P} T_{x}(X)\right)
$$

where $f_{\alpha} \in \mathcal{F}_{\alpha}$ denotes the value of $f$ at $\alpha$, which is contained in $\mathcal{J}_{\alpha}$ so that $\pi_{*} f_{\alpha} \in \hat{\alpha}$.

Proof. We will work on $\Xi$ as in the proof of Proposition 1. Choose a local coordinate system $x_{1}, \ldots, x_{n}$ of $X$ and vertical coordinates $\lambda_{1}=d x_{1}, \ldots, \lambda_{n}=d x_{n}$ of $\Xi$. We choose lifts $\hat{v}=\sum_{i} v_{i} \frac{\partial}{\partial \lambda_{i}}$ and $\hat{u}=\sum_{i} u_{i} \frac{\partial}{\partial \lambda_{i}}$. As in the proof of Proposition 1 , we may choose $\hat{f}=\sum_{i} f_{i} \frac{\partial}{\partial \lambda_{i}}+\sum_{j} \lambda_{j} \frac{\partial}{\partial x_{j}}$. Then

$$
\begin{aligned}
{[\hat{v}, \hat{f}] } & =\sum_{i} v_{i} \frac{\partial}{\partial x_{i}}+\sum_{i}\left(\hat{v}\left(f_{i}\right)-\hat{f}\left(v_{i}\right)\right) \frac{\partial}{\partial \lambda_{i}} \\
{[\hat{u},[\hat{v}, \hat{f}]] } & \equiv \sum_{i} \hat{u}\left(v_{i}\right) \frac{\partial}{\partial x_{i}} \bmod \hat{\mathcal{V}} .
\end{aligned}
$$

Restricting $\hat{u}$ and $\hat{v}$ to $\hat{\mathcal{C}}_{x}$, we see that $[\hat{u},[\hat{v}, \hat{f}]]_{\alpha}=\hat{I I} I_{\alpha}(u, v)$.

Define a sub-distribution $\mathcal{G}$ of $\mathcal{V}$ which consists of tangent vectors to fibers of the Gauss map for $\mathcal{C}_{x}$ as $x$ varies. This distribution is integrable. An immediate consequence of Proposition 2 is

Proposition 3. In the notation above, $\mathcal{G}=C h(\mathcal{P}) \cap \mathcal{V}$.

This enables us to describe $C h(\mathcal{P})$ in terms of the Gauss map for $\mathcal{C}_{x}$ as follows.

Proposition 4. If there exists a complement $\mathcal{F}$ to $\mathcal{V}$ in $\mathcal{J}$ in an open subset of $\mathcal{C}$ so that $\mathcal{F} \subset C h(\mathcal{P})$, then $C h(\mathcal{P})=\mathcal{F}+\mathcal{G}+[\mathcal{F}, \mathcal{G}]$ on that open subset. In this case, if the rank of $\mathcal{G}$ is $k-1$ for some $k>0$, then the rank of $C h(\mathcal{P})$ is $2 k-1$.

Proof. From Proposition 3 and the fact that $C h(\mathcal{P})$ is closed under Lie bracket, the inclusion $\mathcal{F}+\mathcal{G}+[\mathcal{F}, \mathcal{G}] \subset C h(\mathcal{P})$ is immediate. We know that any local section of $C h(\mathcal{P})$ can be written as $f_{1}+v_{1}+\left[f_{2}, v_{2}\right]$ for some local sections $f_{1}, f_{2}$ of $\mathcal{F}$ and $v_{1}, v_{2}$ of $\mathcal{V}$ from Proposition 1 . We want to show that this local section is in $\mathcal{F}+\mathcal{G}+[\mathcal{F}, \mathcal{G}]$. It suffices to show that $v_{2}$ is a section of $C h(\mathcal{P})$. In fact, if $v_{2}$ is a section of $C h(\mathcal{P})$, it is a section of $\mathcal{G}$ from Proposition 3 and so $\left[f_{2}, v_{2}\right]$ is a local section of $[\mathcal{F}, \mathcal{G}]$. As a consequence, we have $v_{1} \in C h(\mathcal{P}) \cap \mathcal{V}=\mathcal{G}$, which proves Proposition 4 . To show that $v_{2}$ is a section of $C h(\mathcal{P})$, we need to check that $\left[v_{2}, h\right]$ is a section of $\mathcal{P}$ for any local 
section $h$ of $\mathcal{P}$. From Proposition 1 , we can set $h=f_{3}+v_{3}+\left[f_{4}, v_{4}\right]$. Then

$$
\begin{aligned}
{\left[v_{2}, h\right] } & =\left[v_{2}, f_{3}+v_{3}+\left[f_{4}, v_{4}\right]\right] \\
& =\left[v_{2}, f_{3}\right]+\left[v_{2}, v_{3}\right]+\left[v_{2},\left[f_{4}, v_{4}\right]\right] \\
& =\left[v_{2}, f_{3}\right]+\left[v_{2}, v_{3}\right]+\left[v_{4},\left[f_{4}, v_{2}\right]\right]+\left[f_{4},\left[v_{2}, v_{4}\right]\right] .
\end{aligned}
$$

So it suffices to show that $\left[v_{4},\left[f_{4}, v_{2}\right]\right]$ is a section of $\mathcal{P}$. Since $f_{1}+v_{1}+\left[f_{2}, v_{2}\right]$ is a section of $C h(\mathcal{P})$, we see that $\left[v_{4}, f_{1}+v_{1}+\left[f_{2}, v_{2}\right]\right]$ is a section of $\mathcal{P}$. Thus $\left[v_{4},\left[f_{2}, v_{2}\right]\right]$ is a section of $\mathcal{P}$. This implies that $\left[v_{4},\left[f_{4}, v_{2}\right]\right]$ is a section of $\mathcal{P}$ because $f_{4}=\zeta f_{2}$ for some local holomorphic function $\zeta$.

Finally, the statement about the ranks follows from the local coordinate expression of $[\mathcal{F}, \mathcal{G}]$ as in the proof of Proposition 1.

Proposition 5. Suppose there exists a local complement $\mathcal{F}$ to $\mathcal{V}$ in $\mathcal{J}$ as in Proposition 4. Let $S$ be a local leaf of $C h(\mathcal{P})$ in an open neighborhood of the domain of definition of $C h(\mathcal{P})$ where $\mathcal{F}$ is a well-defined foliation. Then $S$ is an open subset in $\mathbb{P} T(\pi(S))$.

Proof. Since the leaves of $\mathcal{F}$ in $S$ are sent to holomorphic curves in $\pi(S)$, we see that $S \subset \mathbb{P} T(\pi(S))$. Let $k-1$ be the rank of $\mathcal{G}$ so that $\operatorname{dim} S=2 k-1$. Note that the intersection of $S$ with a fiber $\pi^{-1}(x)$ is an open subset of the projective space in $\mathcal{C}_{x}$ which is a fiber of the Gauss map of $\mathcal{C}_{x}$. Thus we get $\operatorname{dim}(\pi(S))=k$, which implies that $\operatorname{dim}(S)=\operatorname{dim} \mathbb{P} T(\pi(S))$.

3. Birationality of the tangent map. For a quick reference on the deformation theory of rational curves in the terminology of the current article we refer the reader to [HM2]. Let $X$ be a uniruled projective manifold and $\mathcal{K}$ be a minimal component. Let $\rho: \mathcal{U} \rightarrow \mathcal{K}$ and $\mu: \mathcal{U} \rightarrow X$ be the associated universal family morphisms. Since $\tau: \mathcal{U} \rightarrow \mathcal{C}$ is generically finite, there exists a Zariski open subset $\mathcal{U}^{o}$ such that $\left.\tau\right|_{\mathcal{U}}$ is an étale morphism. The fibers of $\rho: \mathcal{U} \rightarrow \mathcal{K}$ can be regarded as a foliation by curves on an étale cover of an open subset of $\mathcal{C}$. This will be called the tautological foliation on $\mathcal{C}$ and denoted by $\mathcal{F}$. This is a multi-valued foliation on $\mathcal{C}$.

Proposition 6. The tautological foliation $\mathcal{F}$ is a univalent foliation on $\mathcal{C}$ if and only if the tangent map $\tau: \mathcal{U} \rightarrow \mathcal{C}$ is birational.

Proof. The univalence of $\mathcal{F}$ when $\tau$ is birational is obvious. If $\mathcal{F}$ is univalent, its leaves are curves on $\mathcal{C}$ whose images in $X$ under the projection $\pi: \mathcal{C} \rightarrow X$ are just members of $\mathcal{K}$. Thus $\tau$ must be birational from the generic injectivity of the natural map $\mathcal{K} \rightarrow \operatorname{Chow}(X)$.

Now let us apply Section 2 to the subvariety $\mathcal{C}$ of $\mathbb{P} T(X)$. We have natural distributions $\mathcal{V}, \mathcal{J}$ and $\mathcal{P}$ on $\mathcal{C}$ which are completely determined by the inclusion $\mathcal{C} \subset \mathbb{P} T(X)$.

Proposition 7. At a general point of $\mathcal{C}$ choose a neighborhood on which a univalent choice of the values of the tautological foliation $\mathcal{F}$ can be made. Denote this univalent foliation by the same symbol $\mathcal{F}$. Then $\mathcal{V}+\mathcal{F}=\mathcal{J}$ in that open subset.

Proof. By the definition of $\mathcal{J}, \mathcal{J}_{\alpha}=(d \pi)^{-1}(\mathbb{C} \alpha)$ at $\alpha \in \mathcal{C}$, it is obvious that $\mathcal{F} \subset$ $\mathcal{J}$. Thus $\mathcal{V}+\mathcal{F}=\mathcal{J}$ follows from $\mathcal{F} \nsubseteq \mathcal{V}$ and $\operatorname{rank}(\mathcal{V})+\operatorname{rank}(\mathcal{F})=\operatorname{rank}(\mathcal{J})$. 
On $\mathcal{K}$, we have a natural distribution $\mathcal{R}$ defined as follows. At a general point $[h]$ of $\mathcal{K}$ corresponding to a rational curve $h: \mathbb{P}^{1} \rightarrow X$, the bundle $h^{*} T(X)$ on $\mathbb{P}^{1}$ splits as $\mathcal{O}(2) \oplus \mathcal{O}(1)^{p} \oplus \mathcal{O}^{n-1-p}$ for some non-negative integer $p$. Members of $\mathcal{K}$ having such splitting type of $T(X)$ are called standard rational curves. By elementary deformation theory, $p$ is the fiber dimension of $\mu: \mathcal{U} \rightarrow X$ and the tangent space to $\mathcal{K}$ is

$$
T_{[h]}(\mathcal{K})=H^{0}\left(\mathbb{P}^{1}, h^{*} T(X)\right) / H^{0}\left(\mathbb{P}^{1}, T\left(\mathbb{P}^{1}\right)\right) \cong H^{0}\left(\mathbb{P}^{1}, \mathcal{O}(1)^{p} \oplus \mathcal{O}^{n-1-p}\right) .
$$

The subspace of $T_{[h]}(\mathcal{K})$ corresponding to the subspace $H^{0}\left(\mathbb{P}^{1}, \mathcal{O}(1)^{p}\right)$ is determined independent of the choice of the isomorphism $h^{*} T(X) \cong \mathcal{O}(2) \oplus \mathcal{O}(1)^{p} \oplus \mathcal{O}^{n-1-p}$. This subspace will be defined to be $\mathcal{R}_{[h]}$. Thus $\mathcal{R}$ is a distribution of rank $2 p$ on $\mathcal{K}$ whose domain of definition includes the open subset consisting of standard rational curves belonging to $\mathcal{K}$.

Proposition 8. The pull-back of $\mathcal{R}$ by $\rho$ agrees with the pull-back of $\mathcal{P}$ by $\tau$ : $d \tau^{*} \mathcal{P}=d \rho^{*} \mathcal{R}$. In particular, $\mathcal{F} \subset C h(\mathcal{P})$.

Proof. At a general point $\alpha \in \mathcal{C}$ corresponding to the tangent direction to a general standard minimal rational curve $h: \mathbb{P}^{1} \rightarrow X$ with $h(o)=x$, we have the natural identifications

$$
\begin{gathered}
T_{\alpha}(\mathcal{C})=H^{0}\left(\mathbb{P}^{1}, h^{*} T(X)\right) / H^{0}\left(\mathbb{P}^{1}, T\left(\mathbb{P}^{1}\right) \otimes \mathbf{m}_{o}\right) \\
T_{x}(X)=H^{0}\left(\mathbb{P}^{1}, h^{*} T(X)\right) / H^{0}\left(\mathbb{P}^{1}, h^{*} T(X) \otimes \mathbf{m}_{o}\right) .
\end{gathered}
$$

Under these identifications, the projection $d \pi: T_{\alpha}(\mathcal{C}) \rightarrow T_{x}(X)$ corresponds to taking the value of a section in $H^{0}\left(\mathbb{P}^{1}, h^{*} T(X)\right)$ at the point $o$. By definition, $\mathcal{P}_{\alpha}=$ $(d \pi)^{-1}\left(\hat{T}_{\alpha}\left(\mathcal{C}_{x}\right)\right)$ at a general point $\alpha \in \mathcal{C}$. By elementary deformation theory, $\hat{T}_{\alpha}\left(\mathcal{C}_{x}\right)$ is naturally isomorphic to $\left(\mathcal{O}(2) \oplus \mathcal{O}(1)^{p}\right)$-part of the splitting $h^{*} T(X) \cong \mathcal{O}(2) \oplus$ $\mathcal{O}(1)^{p} \oplus \mathcal{O}^{n-1-p}$. Thus $\mathcal{P}_{\alpha}$ corresponds to the sections in $H^{0}\left(\mathbb{P}^{1}, h^{*} T(X)\right)$ whose values at $o$ lie in the $\mathcal{O}(2) \oplus \mathcal{O}(1)^{p}$-part of the splitting. Thus it is exactly the lift of $\mathcal{R}_{[h]}$ by $\rho$.

Proposition 9. Let $S$ be a general leaf of $C h(\mathcal{P})$. Then $\pi(S)$ is a quasiprojective variety and contains a smooth Zariski open subset $\mathcal{W} \subset \pi(S)$ such that $\mathbb{P} T(\mathcal{W})$ is a Zariski dense open subset of $S$. Let $\mathcal{K}^{S}$ be the subvariety of $\mathcal{K}$ consisting of members of $\mathcal{K}$ lying on the closure $\bar{S}$ of $\pi(S)$. Then $\mathcal{K}^{S}$ is a minimal component of the irreducible projective variety $\bar{S}$. Moreover, the corresponding total space of minimal rational tangents $\mathcal{C}^{S}$ agrees with $\mathbb{P} T(\bar{S})$.

Proof. By Proposition 7 and Proposition 8, we can apply Proposition 5 here. The result follows from the fact that the leaves of $\mathcal{F}$ correspond to minimal rational curves.

Now we recall the following result of Cho-Miyaoka-Shepherd-Barron. Note that minimal components and their total spaces of minimal rational tangents are defined for any irreducible projective variety $X$ in Section 1.

Proposition 10 ([CMS, TheOrem 4.2]). Let $X$ be an irreducible normal projective variety of dimension $n$. Suppose there exists a minimal component $\mathcal{K}$ on $X$ such that the total space of minimal rational tangents agrees with $\mathbb{P} T(X)$. Then there exists a finite morphism $\mathbb{P}^{n} \rightarrow X$ which is étale over $X-\operatorname{Sing}(X)$ such that the members of $\mathcal{K}$ are just the images of lines in $\mathbb{P}^{n}$. In particular, $X \cong \mathbb{P}^{n}$ if $X$ is smooth. 
A simple consequence is the following special case of Theorem 1.

Proposition 11. Let $X$ be an irreducible normal projective variety which has a minimal component $\mathcal{K}$ such that the total space of minimal rational tangents $\mathcal{C}$ agrees with $\mathbb{P} T(X)$. Then

(i) the tangent map $\tau: \mathcal{U} \rightarrow \mathcal{C}$ is birational and

(ii) $\mathcal{K}$ is the only minimal component of $X$.

We are ready to prove Theorem 1 and 2 .

Proof of Theorem 1 and Theorem 2. Let us start with Theorem 1. By Proposition 6 , it suffices to show that the tautological foliation $\mathcal{F}$ is univalent. By Proposition 8, $\mathcal{F}$ is contained in $C h(\mathcal{P})$ and it suffices to show that it is univalent on a general leaf $S$ of $C h(\mathcal{P})$. But by Proposition $9, \mathcal{F}$ restricted to $S$ is the tautological foliation for the minimal component $\mathcal{K}^{S}$ of $\bar{S}$ with the total space of minimal rational tangents $\mathcal{C}^{S}$ equal to $\mathbb{P} T(\bar{S})$. Thus $\mathcal{F}$ is univalent on $S$ by Proposition 11 (i) applied to the normalization of $\bar{S}$. Theorem 2 follows from the fact that $\mathcal{K}^{S}$ is the only minimal component of $\bar{S}$, by Proposition 11 (ii).

We will finish this section with a few results about $\pi(S)$ in the notation of Proposition 9. A subvariety $Y$ in $X$ is called a Cauchy subvariety of the minimal component $\mathcal{K}$ if it is the closure of $\pi(S)$ for a general leaf $S$ of $C h(\mathcal{P})$ in the notation of Proposition 9. The following is an immediate consequence of Proposition 10.

Proposition 12. Let $X$ be a uniruled projective manifold and $Y \subset X$ be a Cauchy subvariety with respect to a choice of a minimal component $\mathcal{K}$. Let $\tilde{Y}$ be the normalization of $Y$. Then there exists a finite morphism $\mathbb{P}^{d} \rightarrow \tilde{Y}$ which is étale over $\tilde{Y}-\operatorname{Sing}(\tilde{Y})$ such that the members of $\mathcal{K}$ lying on $Y$ are just the images of lines in $\mathbb{P}^{d}$.

Corollary 1 has the following consequence.

Proposition 13. Let $X$ be a uniruled projective manifold and $\mathcal{K}$ be a minimal component. Suppose the variety of minimal rational tangents $\mathcal{C}_{x}$ is non-linear. Then for each component $\mathcal{C}_{x}^{1}$ of $\mathcal{C}_{x}$, the intersection of the closures of the fibers of the Gauss map for $\mathcal{C}_{x}^{1}$ is empty.

Proof. Suppose there exists a point $\alpha \in \mathcal{C}_{x}^{1}$ which is the intersection of the closures of the fibers of the Gauss map. The normalization $\mathcal{K}_{x}^{1}$ of $\mathcal{C}_{x}^{1}$ is smooth by Corollary 1. The fibers of the Gauss map give subvarieties of the smooth projective variety $\mathcal{K}_{x}^{1}$ passing through a point $\hat{\alpha} \in \mathcal{K}_{x}^{1}$ over $\alpha$. The pull-back of $\mathcal{O}(1)$ bundle on $\mathbb{P} T_{x}(X)$ to $\mathcal{K}_{x}^{1}$ gives an ample line bundle $L$ with respect to which the fibers of the Gauss map are linear subspaces. It follows that there exists a family of rational curves through $\hat{\alpha}$ covering the whole smooth variety $\mathcal{K}_{x}^{1}$ such that each member has degree 1 with respect to the ample line bundle $L$. By Proposition $10, \mathcal{K}_{x}^{1}$ must be a projective space and then $\mathcal{C}_{x}^{1}$ must be linear.

It is well-known that a positive-dimensional family of minimal rational curves passing through a general point $x \in X$ does not have a common intersection other than $x$ ('bend-and-break'). A weaker form of this fact can be generalized to Cauchy subvarieties as follows. 
Proposition 14. Let $X$ be a uniruled projective manifold and $\mathcal{K}$ be a minimal component. Assume that $\mathcal{C}_{x}$ is non-linear for a general point $x \in X$. Let $\mathcal{C}_{x}^{1}$ be a component of $\mathcal{C}_{x}$. Then the irreducible family of the Cauchy subvarieties passing through $x$ whose tangent spaces lie in $\mathcal{C}_{x}^{1}$ does not have a common intersection point other than $x$.

Proof. Note that the non-linearity of $\mathcal{C}_{x}$ implies, by Lemma 2, that there is a positive-dimensional family $\left\{Y_{s}\right\}$, where $s$ is a parameter, of Cauchy subvarieties passing through $x$ whose tangent spaces lie in $\mathcal{C}_{x}^{1}$. Suppose there exists a common intersection point $y$ different from $x$. By Proposition 12, for each $s$, there exists a member $C_{s}$ of $\mathcal{K}$ passing through $x$ and $y$ lying on the Cauchy subvariety $Y_{s}$. Since there cannot exist a positive-dimensional family of minimal rational curves passing through $x$ and $y, C_{s}$ cannot be a family of distinct members of $\mathcal{K}$. It follows that a single member $C=C_{s}$ belongs to each $Y_{s}$ and $\alpha:=\mathbb{P} T_{x}\left(C_{s}\right)$ is in the intersection of the fibers of the Gauss map of $\mathcal{C}_{x}^{1}$, a contradiction to Proposition 13.

4. Rigidity of generically finite morphisms to Fano manifolds with nonlinear varieties of minimal rational tangents. A weaker form of Theorem 3 was already proved in [HM3] where the stronger assumption that $\mathcal{C}_{x}$ has generically finite Gauss map was needed. The proof of Theorem 3 is a generalization of the argument in [HM3] using the result of Section 3. We start with recalling two results from [HM3].

Lemma 3 ([HM3, Lemma 4.2]). Let $\pi: Y \rightarrow X$ be a generically finite morphism from a normal variety $Y$ onto a Fano manifold $X$ with Picard number 1 . Suppose for a general member $C \subset X$ belonging to a chosen minimal component $\mathcal{K}$, each component of the inverse image $\pi^{-1}(C)$ is birational to $C$ by $\pi$. Then $\pi: Y \rightarrow X$ itself is birational.

Proposition 15 ([HM3, Proof of Theorem 1.4]). Let $\left\{X_{t}, t \in \Delta\right\}$ be $a$ regular family of uniruled projective manifolds. Given a minimal component $\mathcal{K}_{0}$ of $X_{0}$ with the total space of variety of minimal rational tangents $\mathcal{C}_{0} \subset \mathbb{P} T\left(X_{0}\right)$, there exists $\epsilon>0$ and a family of minimal components $\mathcal{K}_{t}$ of $X_{t}, 0<|t|<\epsilon$, with the total space of minimal rational tangents $\mathcal{C}_{t} \subset \mathbb{P} T\left(X_{t}\right)$ having the following property: given a family of generically finite morphisms $f_{t}: X^{\prime} \rightarrow X_{t}$ from a fixed projective manifold $X^{\prime}$ and a general point $x \in X^{\prime}$, the family of subvarieties $\left(d f_{t}\right)_{x}^{-1}\left(\mathcal{C}_{t, f_{t}(x)}\right)$ in $\mathbb{P} T_{x}\left(X^{\prime}\right)$ is a constant family, i.e.

$$
\left(d f_{t}\right)^{-1}\left(\mathcal{C}_{t, f_{t}(x)}\right)=\left(d f_{0}\right)^{-1}\left(\mathcal{C}_{0, f_{0}(x)}\right)
$$

for all $|t|<\epsilon$.

The condition of the non-linearity of the variety of minimal rational tangents is used in the following manner.

Proposition 16. Let $X$ be a Fano manifold of Picard number 1. Suppose there exists a minimal component $\mathcal{K}$ such that the variety of minimal rational tangents $\mathcal{C}_{x}$ at a general point $x \in X$ is non-linear of dimension $p>0$. Let $X^{\prime}$ be another Fano manifold of Picard number 1 with a minimal component $\mathcal{K}^{\prime}$. Assume that the variety of minimal rational tangents $\mathcal{C}_{x^{\prime}}^{\prime} \subset \mathbb{P} T_{x^{\prime}}\left(X^{\prime}\right)$ at a general point $x^{\prime}$ has dimension $p$. If there exists a quasi-projective variety $U$ and étale morphisms $e: U \rightarrow X$ and $\varphi: U \rightarrow$ $X^{\prime}$ preserving varieties of minimal rational tangents in the sense that $\varphi_{*} e^{*}\left(\mathcal{C}_{e(y)}\right)=$ 
$\mathcal{C}_{\varphi(y)}^{\prime}$ (and hence $\left.e_{*} \varphi^{*}\left(\mathcal{C}_{\varphi(y)}^{\prime}\right)=\mathcal{C}_{e(y)}\right)$ for all $y \in U$, then there exists a birational map $\Phi: X \rightarrow X^{\prime}$ such that $\varphi=\Phi \circ e$.

Proof. We define an equivalence relation on $U$ by $y_{1} \sim y_{2}$ if $e\left(y_{1}\right)=e\left(y_{2}\right)$ and $\varphi\left(y_{1}\right)=\varphi\left(y_{2}\right)$. In the quotient space $U / \sim$, we can find a Zariski-open subset $\tilde{U}$ so that the induced morphisms $\tilde{e}: \tilde{U} \rightarrow X$ and $\tilde{\varphi}: \tilde{U} \rightarrow X^{\prime}$ are étale, preserving varieties of minimal rational tangents in the above sense. For a general Cauchy subvariety $Y$ of $\mathcal{K}$, any component $Y_{1}$ of $\tilde{e}^{-1}(Y)$ is sent to a Cauchy subvariety of $\mathcal{K}^{\prime}$ because Cauchy subvarieties are completely determined by the geometry of the total space of minimal rational tangents in $\mathbb{P} T(X)$ and $\mathbb{P} T\left(X^{\prime}\right)$.

We claim that $\tilde{e}$ is injective. Suppose not. Then by Lemma 3 , for a general member $C$ of $\mathcal{K}$, there exists a component $\hat{C}$ of $\tilde{e}^{-1}(C)$ so that $\left.\tilde{e}\right|_{\hat{C}}: \hat{C} \rightarrow C$ is not birational. Choose a general point $x \in C$ and let $y_{1} \neq y_{2}$ be points of $\hat{C}$ over $x$. By the construction of $\tilde{U}$ we can assume that $\tilde{\varphi}\left(y_{1}\right) \neq \tilde{\varphi}\left(y_{2}\right)$. Since $C$ is general, we can assume that for $p$-dimensional family of deformations $C_{t}$ of $C$ fixing $x$, the collection of some components $\hat{C}_{t}$ of $\tilde{e}^{-1}\left(C_{t}\right)$ gives a $p$-dimensional family of deformations of $\hat{C}$ fixing $y_{1}$ and $y_{2}$. For the Cauchy subvariety $S_{t}$ containing $C_{t}, \tilde{e}^{-1}\left(S_{t}\right)$ contains an irreducible component $Z_{t}$ containing both $y_{1}$ and $y_{2}$. Thus we have a component $\mathcal{C}^{1}$ of the variety of minimal rational tangents $\mathcal{C}_{\tilde{\varphi}\left(y_{1}\right)}^{\prime}$ and a component $\mathcal{C}^{2}$ of the variety of minimal rational tangents $\mathcal{C}_{\tilde{\varphi}\left(y_{2}\right)}^{\prime}$ such that the Cauchy subvarieties corresponding to $\mathcal{C}^{1}$ and the Cauchy subvarieties corresponding to $\mathcal{C}^{2}$ are equal. Since $\mathcal{C}_{x^{\prime}}^{\prime} \subset \mathbb{P} T_{x^{\prime}}\left(X^{\prime}\right)$ is also non-linear at a general point $x^{\prime} \in X^{\prime}$, this contradicts with Proposition 14.

Proof of Theorem 3. Suppose that $f$ is birational. Then $f$ is biholomorphic over $\mathcal{X}-\mathcal{Z}$ where $\mathcal{Z}$ is a subvariety of codimension $\geq 2$. On $\mathcal{X}^{\prime}$ we have the vector field $\mathcal{V}$ lifting $\frac{d}{d t}$ on $\Delta$. Its push-forward $f_{*} \mathcal{V}$ is a vector field on $\mathcal{X}-\mathcal{Z}$. By Hartogs, we can extend it to a vector field on $\mathcal{X}$ which generates the required family of biholomorphic morphisms.

Suppose that $f_{t}$ is not birational, but generically $d$-to- 1 . We will construct a new projective manifold $\hat{X}$, a generically finite dominant rational map $\nu: X^{\prime}-\hat{X}$ of degree $d$ and a holomorphic family of birational morphisms $g_{t}: \hat{X} \rightarrow X_{t}$ for small $t$, so that $f_{t}=g_{t} \circ \nu$ over general points of $X^{\prime}$. Then the proof is reduced to the birational case, which is already settled above.

Let $\mathcal{K}^{0}$ be the minimal component on $X_{0}$ with the total space of minimal rational tangents $\mathcal{C}^{0} \subset \mathbb{P} T\left(X_{0}\right)$ whose fiber over a general point of $X_{0}$ consists of non-linear subvarietes. Applying Proposition 15, there exists some $\epsilon>0$ and a minimal component $\mathcal{K}_{t}$ of $X_{t}$ for $0<|t|<\epsilon$ such that the total space of minimal rational tangents $\mathcal{C}^{t}$ satisfies

$$
\left(d f_{t}\right)_{y}^{-1}\left(\mathcal{C}_{f_{t}(y)}^{t}\right)=\left(d f_{0}\right)_{y}^{-1}\left(\mathcal{C}_{f_{0}(y)}^{0}\right)
$$

Fix such a small $t=s$. There exists an open subset $U \subset X^{\prime}$ which is étale over $X_{0}$ and $X_{s}$ by $f_{0}$ and $f_{s}$. The above equality of the inverse image of the varieties of minimal rational tangents at $f_{s}(y)$ and $f_{0}(y)$ implies that the hypothesis of Proposition 16 is satisfied for the étale morphisms $\left.f_{s}\right|_{U}$ and $\left.f_{0}\right|_{U}$. Thus there exists a birational $\operatorname{map} \Phi: X_{0} \rightarrow X_{s}$ such that $\left.f_{s}\right|_{U}=\Phi \circ f_{0} \mid U$.

We say that a reduced 0 -cycle $y_{1}+\cdots+y_{d}$ of length $d$ on $X^{\prime}$ is a special cycle if $f_{0}\left(y_{1}\right)=\cdots=f_{0}\left(y_{d}\right)$ and $f_{s}\left(y_{1}\right)=\cdots=f_{s}\left(y_{d}\right)$. From the existence of the birational map $\Phi$, general fibers of $f_{0}$ and $f_{s}$ are special cycles. The set of all special cycles on 
$X^{\prime}$ gives a constructible subset of the Hilbert scheme of 0 -dimensional subschemes of $X^{\prime}$. We can find an irreducible component of this set so that the corresponding cycles cover an open set of $X^{\prime}$. Let $B$ be the closure of that component and let $\sigma: A \rightarrow B$ and $\lambda: A \rightarrow X^{\prime}$ be the universal family morphisms so that $\sigma$ is flat and of degree $d$. We claim that $\lambda$ is birational. Otherwise we have two distinct special cycles of degree $d$ containing a given general point $y$ of $X^{\prime}$, which is absurd because $f_{0}$ and $f_{s}$ are of degree $d$.

Let $\hat{X}$ be a desingularization of $B$ and $\hat{\sigma}: \hat{A} \rightarrow \hat{X}, \hat{\lambda}: \hat{A} \rightarrow X^{\prime}$ be the induced morphisms. Define the rational map $\nu: X^{\prime} \rightarrow \hat{X}$ as $\nu:=\hat{\sigma} \circ \hat{\lambda}^{-1}$. Then $\nu$ is a generically finite dominant rational map of degree $d$. Consider the morphism $\hat{f}_{s}=f_{s} \circ \hat{\lambda}$ from $\hat{A}$ to $X_{s}$. From the definition of special cycles, a general fiber of $\hat{\sigma}$ is contained in a fiber of $\hat{f}_{s}$. Thus each fiber of $\hat{\sigma}$ is contained in a fiber of $\hat{f}_{s}$ by the flatness of $\hat{\sigma}$. It follows that we get a birational morphism $g_{s}: \hat{X} \rightarrow X_{s}$ satisfying $f_{s}=g_{s} \circ \nu$. Since $X^{\prime}, X_{s}$ are all projective, it is easy to see that $\left\{g_{s}\right\}$ is a holomorphic family.

Let $X$ be a Fano manifold of Picard number 1. Suppose $X^{\prime}$ is a projective manifold with non-zero holomorphic vector fields. Given a generically finite morphism $f: X^{\prime} \rightarrow X$, we get a deformation $f_{t}: X^{\prime} \rightarrow X$ of $f$ obtained by composing it with the 1-parameter subgroup of automorphisms of $X^{\prime}$ generated by the holomorphic vector fields. In this situation, Theorem 3 , with $\mathcal{X}=X \times \Delta$, implies the following.

Corollary 2. Let $X$ be a Fano manifold of Picard number 1 which has a minimal component with non-linear variety of minimal rational tangents. Then for any projective variety $X^{\prime}$ and a surjective generically finite morphism $f: X^{\prime} \rightarrow X$, any holomorphic vector field on $X^{\prime}$ descends to a holomorphic vector field on $X$ such that $f$ is equivariant with respect to the 1-parameter groups of automorphisms of $X^{\prime}$ and $X$ generated by the holomorphic vector fields.

Proof of Theorem 4. Since the Picard number of $G / P$ is $1, f$ is finite and $X$ is a Fano manifold of Picard number 1. Assume that $X \neq \mathbb{P}_{n}$ and pick a minimal component $\mathcal{K}$ on $X$ with $\mathcal{C} \neq \mathbb{P} T(X)$ by Proposition 10 .

First assume that $\mathcal{C}_{x}$ is non-linear at general $x \in X$. By Corollary 2, vector fields on $G / P$ descend to vector fields on $X$ by $f$. If $R \subset G / P$ is a non-empty ramification divisor of $f$ and $B=f(R)$, then the image of any tangent vector of $G / P$ at a point $x$ on $R$ is tangent to $B$ whenever $f(x)$ is a smooth point of $B$. Thus all vector fields of $G / P$ are sent to vector fields on $X$ tangent to $B$ at smooth points of $B$. This implies that integral curves of any vector field of $G / P$ through a point of $R$ are sent to curves in $B$. In other words, the integral curves through a point of $R$ remain in $R$, a contradiction to the homogeneity of $G / P$.

Now we may assume that $\mathcal{C}_{x}$ is linear. Then a component of $(d f)^{-1}\left(\mathcal{C}_{f(s)}\right) \subset$ $\mathbb{P}_{s}(G / P)$ at a general point $s \in G / P$ is a linear space invariant under the isotropy group $P$ by [HM1, Section 1], defining a $G$-invariant distribution on $G / P$. By translating an irreducible component of the inverse image of a Cauchy subvariety, we get a proper $G$-invariant foliation on $G / P$ with compact leaves, a contradiction to the assumption that $G / P$ has Picard number 1 . 


\section{REFERENCES}

[CMS] Cho, K., Miyaoka, Y. And Shepherd-Barron, N. I., Characterization of projective space and applications to complex symplectic manifolds, in Higher dimensional birational geometry (Kyoto, 1997), Adv. Stud. Pure Math., 35 (2002), pp. 1-88.

[GH] Griffiths, P. And Harris, J., Algebraic geometry and local differential geometry, Ann. scient. Éc. Norm. Sup., 12 (1979), pp. 355-432.

[HM1] HWANG, J.-M. AND MoK, N., Holomorphic maps from rational homogeneous spaces of Picard number 1 onto projective manifolds, Invent. math., 136 (1999), pp. 209-231.

[HM2] HwANG, J.-M. AND MOK, N., Varieties of minimal rational tangents on uniruled manifolds, in Several Complex Variables, ed. by M. Schneider and Y.-T. Siu, MSRI Publications 37, Cambridge University Press (1999), pp. 351-389.

[HM3] Hwang, J.-M. AND Mok, N., Cartan-Fubini type extension of holomorphic maps for Fano manifolds of Picard number 1, Journal Math. Pures Appl., 80 (2001), pp. 563-575.

[Ke] Kebekus, S., Families of singular rational curves, J. Algebraic Geom., 11 (2002), pp. 245256.

[KK] Kebekus, S. And Kovacs, S., Are minimal degree rational curves determined by their tangent vectors?, preprint alg-geom/0206193.

[Ko] Kollár, J., Rational curves on algebraic varieties, Ergebnisse der Mathematik und ihrer Grenzgebiete, 3 Folge, Band 32, Springer Verlag, 1996. 
J.-M. HWANG AND N. MOK 